\title{
Ứng dụng BIM trong đào tạo kiến trúc tại trường Đại học Xây dựng Miền Tây
}

\author{
Nguyễn Thị Tâm Đan ${ }^{1}$ \\ ${ }^{1}$ Khoa Kiến trúc, Trường ĐHXD Miền Tây
}

\section{TỪ KHOÁ}

Mô hình thông tin công trình (BIM) Phần mềm BIM

Đào tạo BIM

\section{KEYWORDS}

Building Information Modeling (BIM) BIM software

BIM Education

\section{TÓM TẮT}

Trong bối cảnh công nghệ BIM đang được áp dụng rộng rãi trong ngành xây dựng trên thế giới và ở Việt Nam thì việc đào tạo nguồn nhân lực BIM là vấn đề cấp thiết hiện nay. Bài viết này trình bày tổng quan về việc ứng dụng BIM trong đào tạo và đề xuất một số giải pháp nhằm đẩy mạnh ứng dụng BIM trong đào tạo ngành kiến trúc tại trường Đại học Xây dựng Miền Tây.

\section{1. Đặt vấn đề}

BIM là một khuynh hướng mới đang được các nước tiên tiến trên thế giới áp dụng trong ngành Xây dựng Kiến trúc. Tại Việt Nam, BIM đang được triển khai và sẽ được áp dụng rộng rãi trong hoạt động thiết kế, xây dựng và quản lý vận hành công trình kể từ năm 2021. Tuy nhiên, việc thiếu nguồn nhân lực có khả năng đi theo khuynh hướng BIM là một trở ngại lớn cho việc áp dụng BIM hiện nay. Do đó, vấn đề đặt ra cho các cơ sở đào tạo đại học ngành Xây dựng - Kiến trúc là phải đào tạo được nguồn nhân lực đáp ứng yêu cầu về năng lực sử dụng BIM trong các hoạt động của ngành. Nghiên cứu này thông qua việc tổng kết kinh nghiệm ứng dụng BIM trong đào tạo của các trường đại học trên thế giới và Việt Nam để từ đó đề xuất giải pháp đẩy mạnh việc ứng dụng BIM trong đào tạo ngành kiến trúc tại trường ĐHXD Miền Tây.

\section{Công nghệ BIM}

Theo tiêu chuẩn quốc tế về BIM ISO 19650, BIM được hiểu là Building Information Modeling - Tạo lập mô hình thông tin công trình. Đó là việc sử dụng chung một mô tả dạng số hoá sao cho tiến trình thiết kế, xây dựng và vận hành sản sinh ra được những cơ sở đáng tin cậy nhằm phục vụ cho việc hình thành các quyết định liên quan đến một công trình xây dựng [1].

BIM đã mang lại những thay đổi mang tính cách mạng so với phương pháp làm việc truyền thống về tiến trình làm việc, cách tổ chức quản lý, khai thác thông tin công trình và cách giao tiếp giữa các thành viên dự án.

BIM làm thay đổi tiến trình làm việc của các thành viên dự án (chủ đầu tư, kiến trúc sư, kỹ sư, nhà thầu...). Từ làm việc theo tuần tự truyền thống chuyển sang làm việc tích hợp, với sự tham gia của nhiều bộ môn ngay từ giai đoạn đầu của dự án. Sự chuyển đổi này nhằm nâng cao chất lượng, giảm giá thành, giảm chi phí bảo trì và chi phí vận hành công trình.

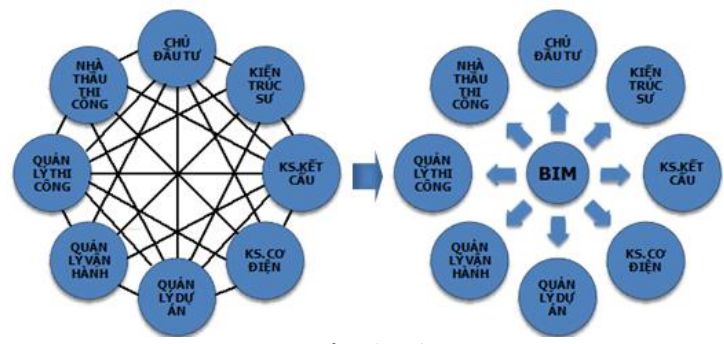

Hình 1. Sự thay đổi về tiến trình làm việc.

Với BIM, cách tổ chức quản lý và khai thác thông tin công trình có sự thay đổi lớn. Toàn bộ thông tin và dữ liệu liên quan đến cả vòng đời của công trình được lưu trữ và khai thác thông qua mô hình thông tin. Mô hình thông tin trở thành sản phẩm quan trọng của giai đoạn thiết kế chứ không phải bản vẽ như phương pháp truyền thống. Bản vẽ chỉ là kết quả của tiến trình khai thác mô hình.
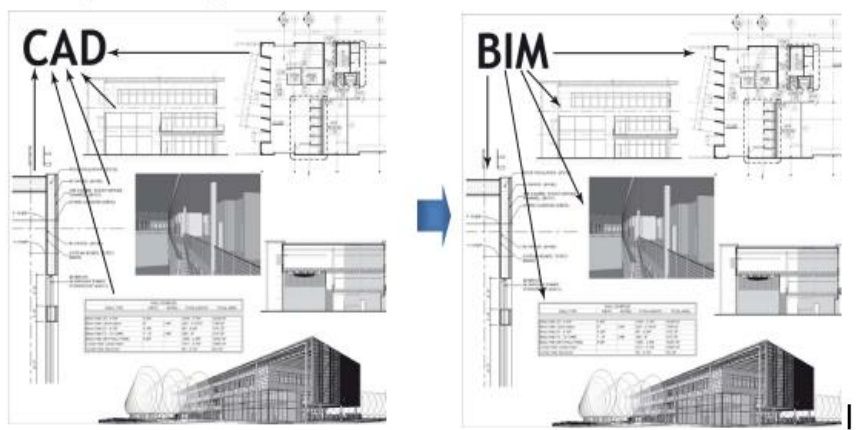

Hình 2. Sự thay đổi về cách tổ chức quản lý \& khai thác thông tin [2]. 
BIM cũng được xem là một công cụ giao tiếp hiệu quả giữa các thành viên dự án. Trong quá trình thực hiện dự án, các thành viên phải chia sẻ và sử dụng thông tin từ các thành viên khác thì mới có thể hoàn thành công việc của mình. Việc sử dụng BIM để quản lý và chia sẻ thông tin về công trình giúp tăng cường sự cộng tác, phối hợp giữa các thành viên của dự án, giúp đưa ra các phương án thiết kế, thi công phù hợp với yêu cầu của chủ đầu tư ngay từ giai đoạn đầu của thiết kế, hạn chế phải làm lại tốn thời gian, tốn chi phí.

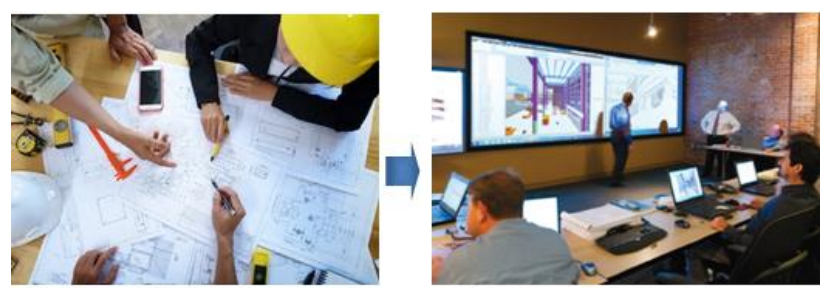

Hình 3. Sự thay đổi về cách giao tiếp giữa các thành viên dự án.

\section{Tình hình ứng dụng BIM trong đào tạo ở các trường đại học trên} thế giới và Việt Nam

3.1. Ứng dụng BIM trong đào tạo ở các trường đại học trên thế giới

Trong những năm qua, các trường đại học trên thế giới không ngừng đẩy mạnh ứng dụng BIM trong đào tạo ngành Xây dựng Kiến trúc với các mức độ khác nhau:

Mỹ: Các trường đại học ở Mỹ đi tiên phong trong việc đào tạo BIM, chiếm đa số trong các trường có đào tạo BIM trên thế giới. Ngoại trừ Viện Công nghệ Georgia nghiên cứu BIM từ đầu những năm 90 , hầu hết các trường đại học ở Mỹ đã bắt đầu giới thiệu BIM kể từ năm 2003.

Từ 2003 - 2005: các trường đại học ở Mỹ chủ yếu giới thiệu về BIM, dạy phần mềm BIM (Đại học Texas $\mathrm{A} \& \mathrm{M}$, Cao đẳng Kỹ thuật Madison, Viện bách khoa Worcester, Đại học California, Đại học Nevada).

Năm 2006, đồ án thiết kế tích hợp sử dụng BIM lần đầu được giới thiệu ở đại học bang Pennsylvania. Sau đó, đại học Auburn, Đại học Wyoming cũng bắt đầu tổ chức cho các sinh viên cùng ngành thực hiện một đồ án cộng tác theo nhóm nhiều sinh viên.

Từ 2007, nhiều trường bắt đầu tổ chức các khóa học cộng tác BIM cho sinh viên đến từ 2 hoặc nhiều ngành ở cùng trường nhằm phát triển kỹ năng cộng tác cho sinh viên (Đại học Pennsylvania, Oklahoma, Auburn, Cal Poly, Texas A\&M, Maryland và Viện Công nghệ Georgia).

Năm 2008, một số trường bắt đầu tổ chức khóa học cộng tác từ xa trên BIM cho sinh viên đến từ 2 hoặc nhiều trường như khóa học Đồ án thiết kế với sự cộng tác của sinh viên đến từ Đại học Wyoming, Đại học bang Montana và Đại học Nebraska -Lincoln.

Ngày nay, tất cả các trường đại học có ngành Xây dựng kiến trúc ở Mỹ đều cung cấp các khóa học về BIM trong chương trình đào tạo. Các trường như Đại học bang Pennsylvania, Viện công nghệ Georgia, Đại học California, Đại học Oklahoma, Đại học Brigham Young, Đại học Purdue là những trường đi đầu trong lĩnh vực đào tạo BIM ở Mỹ $[3,4]$.

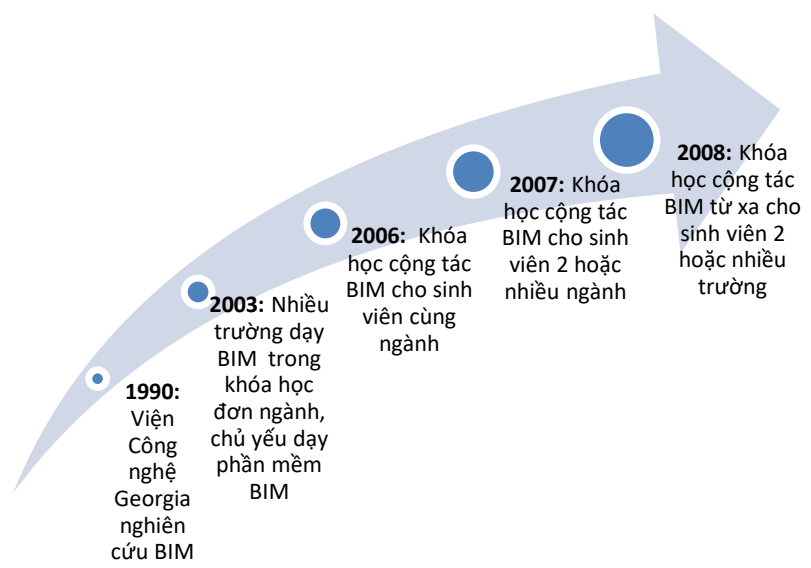

Hình 4. Quá trình phát triển của việc ứng dụng BIM trong đào tạo đại học ở Mỹ.

Anh: Các trường đại học ở Anh cũng đẩy mạnh đưa BIM vào chương trình giảng dạy, chủ yếu ở bậc sau đại học. Các trường đại học như Westminster, Middlesex, Salford, Liverpool, West of England, Northumbria, South Wales cung cấp các khóa học sau đại học chuyên về BIM ở cả hình thức đào tạo chính quy và đào tạo từ xa. Trọng tâm của các khóa học BIM được giới thiệu ở Anh là: Lý thuyết và ứng dụng BIM, Quản lý BIM, BIM và Thiết kế tích hợp, BIM và Sự bền vững [4,5].

Úc: 24 trường đại học tuyên bố có ứng dụng BIM trong chương trình đào tạo, dưới dạng các môn học chuyên về BIM hoặc đưa BIM vào nội dung của các môn học có liên quan. Tuy nhiên, chỉ có 5 trường cung cấp chương trình chuyên về BIM ở bậc đại học và sau đại học: Đại học Bond và Đại học Tây Úc cấp bằng sau đại học về BIM, Đại học Canberra, Đại học Melbourne và Swinburne có chuyên ngành về BIM cấp độ đại học [4].

Phần Lan: tất cả sinh viên ngành Xây dựng Kiến trúc đều được học BIM ở một mức độ nào đó trong chương trình đào tạo. Ở các trường đại học Phần Lan, trọng tâm chính của việc ứng dụng BIM là thiết kế dựa trên BIM trong các lĩnh vực khác nhau. Một số trường cũng cấp bằng cử nhân kiến trúc với lĩnh vực chính yếu là tạo lập và sử dụng mô hình BIM [4].

Đức: Số lượng các trường đại học ở Đức cung cấp các khóa học BIM đã tăng lên trong những năm gần đây, cả ở bậc đại học và sau đại học. Tuy nhiên ở Đức chỉ có các khóa học riêng lẻ về BIM được đưa vào chương trình đào tạo của các trường đại học. Nói chung, việc ứng dụng BIM trong đào tạo ở Đức tập trung vào sử dụng phần mềm BIM, chưa có chương trình đào tạo mới với BIM là nội dung chủ đạo [4].

Cộng hòa Czech: Các trường đại học kỹ thuật hàng đầu của Czech triển khai ứng dụng BIM vào chương trình đào tạo trong vài năm qua, chủ yếu dưới dạng các môn học riêng về BIM hoặc cập nhật BIM trong các môn học có sẳn. Hiện nay, các chương trình đào tạo mới tập trung vào BIM đang nổi lên ở Czech như đại học Ostrava có chương trình sau đại học nhấn mạnh sự phối hợp trong tiến trình xây dựng với BIM [4]. 
Singapore: có 11 cơ sở giáo dục đại học cung cấp các khóa học về BIM/ Thực tế ảo/Thiết kế tích hợp. Phần mềm BIM được chú trọng trong đào tạo. Đến nay, gần 17200 sinh viên và chuyên gia đã được đào tạo về BIM/Thực tế ảo/Thiết kế tích hợp [4].

\section{2. Ứng dụng BIM trong đào tạo ở các trường đại học Việt Nam}

Hiện nay, các trường đại học ở Việt Nam đã rất tích cực trong việc nghiên cứu, triển khai đào tạo BIM, có khá nhiều các chương trình đào tạo đã được xây dựng. Tuy nhiên, phần lớn các chương trình này vẫn chủ yếu là đào tạo về các công cụ BIM, còn thiếu nhiều nội dung liên quan quy trình BIM [6].

Nhằm đảm bảo tính thống nhất về nội dung đào tạo, Bộ Xây dựng đã công bố chương trình khung đào tạo, bồi dưỡng áp dụng Mô hình thông tin công trình (BIM) theo Quyết định số 1056/QĐ-BXD để các cơ quan, tổ chức có liên quan sử dụng trong quá trình thực hiện tổ chức đào tạo, bồi dưỡng các nội dung về BIM. Trên cơ sở chương trình khung này, các trường có thể xây dựng chương trình đào tạo BIM cho phù hợp từng ngành [7].

\section{4. Định hướng tiếp cận ửng dụng BIM trong đào tạo ngành kiến trúc}

Qua tổng kết kinh nghiệm ứng dụng BIM trong đào tạo tại các trường đại học ở trên thế giới và Việt Nam, có thể nhận thấy việc đưa BIM vào chương trình đào tạo được thực hiện theo nhiều phương pháp và hình thức khác nhau. Chương trình đào tạo ngành kiến trúc dẫn đầu về ứng dụng BIM so với các ngành khác [8].

Về trình độ đào tạo: đưa BIM vào chương trình đào tạo ở cả bậc đại học và sau đại học.

Về hình thức đào tạo: tổ chức đào tạo BIM theo hình thức đào tạo chính quy tại trường và đào tạo từ xa.

Về cấp độ ứng dụng BIM trong chương trình đào tạo: đưa BIM vào chương trình đào tạo có 3 cấp độ khác nhau, mỗi cấp độ sẽ có tác động đến cấu trúc chương trình, đội ngũ giảng viên và cơ sở hạ tầng $[9,10]$.

Cấp độ 1: Một số khóa học hiện hữu trong chương trình đào tạo được chọn để kết hợp thêm về BIM. Cấu trúc chương trình, cơ sở vật chất gần như không thay đổi. Giảng viên phụ trách học phần cần hiểu về BIM và tác động của nó.

Cấp độ 2: Bổ sung một số khóa học chuyên về BIM vào chương trình đào tạo. Cấu trúc chương trình đào tạo có sự thay đổi ít. Cơ sở vật chất cần được đầu tư về phòng máy, không gian phù hợp cho việc học. Tất cả giảng viên cần có kiến thức về BIM và hiểu tác động của nó nhưng cần một số giảng viên có đầy đủ năng lực về sử dụng BIM để giảng dạy.

Cấp độ 3: Chương trình đào tạo được xây dựng mới với BIM là nội dung chủ đạo. Cấu trúc chương trình được xem xét toàn diện để BIM trở thành phương tiện chính của việc dạy và học. Cơ sở vật chất cần được đầu tư về phòng máy, không gian đầy đủ cho việc học. Tất cả giảng viên đều hiểu về BIM và tác động của nó, đồng thời có đầy đủ năng lực về sử dụng BIM.
$70 \%$ trường đại học đưa BIM vào chương trình đào tạo dưới dạng kết hợp BIM khóa học hiện hữu hoặc bổ sung các khóa học chuyên về BIM (cấp độ 1\&2), 30 \% trường đại học xây dựng được chương trình đào tạo mới lấy BIM làm trọng tâm (cấp độ 3) [9].

Về hình thức tổ chức giảng dạy: có 3 hình thức tổ chức giảng dạy BIM là khóa học đơn ngành, khóa học liên ngành và cộng tác từ xa [3,9].

Khóa học đơn ngành: tổ chức giảng dạy BIM cho sinh viên cùng ngành trong trường.

Khóa học liên ngành: tổ chức giảng dạy mô phỏng cộng tác BIM cho sinh viên đến từ 2 hoặc nhiều ngành trong trường, dưới dạng đồ án thiết kế tich hợp.

Khóa học cộng tác từ xa: là tổ chức giảng dạy mô phỏng cộng tác BIM cho sinh viên 2 hoặc nhiều ngành đến từ nhiều trường, cũng dưới dạng đồ án thiết kế tich hợp.

Trong 3 hình thức này, các khóa học đơn ngành được tổ chức nhiều hơn 2 hình thức còn lại với khoảng $70 \%$ là các khóa học đơn ngành, $27 \%$ là các khóa học liên ngành, chỉ 3 \% là khóa học cộng tác từ xa [9].

Về phần mềm BIM: Revit Architecture là phần mềm BIM được giảng dạy phổ biến trong chương trình đào tạo ngành kiến trúc với hơn 70 \% các trường đại học ở Mỹ và $79 \%$ các trường đại học ở Anh sử dụng Revit Architecture [11].

Về nội dung đào tạo: nội dung của các khóa học liên quan BIM trong chương trình đào tạo ngành kiến trúc thường bao gồm kiến thức chung về BIM và các kiến thức để phát triển kỹ năng áp dụng BIM như $[8,11]$ :

- Kiến thức, kỹ năng lập mô hình.

- Kiến thức, kỹ năng phân tích thiết kế.

- Kiến thức, kỹ năng phối hợp, cộng tác.

Đây cũng là các nội dung có trong chương trình khung đào tạo, bồi dưỡng áp dụng BIM của Bộ Xây dựng [7].

5. Đề xuất giải pháp đẩy mạnh ứng dụng BIM trong đào tạo ngành kiến trúc tại trường ĐHXD Miền Tây

5.1. Thực trạng ứng dụng BIM trong đào tạo ngành kiến trúc

Trường ĐHXD Miền Tây bắt đầu đào tạo ngành kiến trúc từ năm 2012, đến nay đã có 4 khóa tốt nghiệp (khóa 2012-2015). Trong chương trình đào tạo những năm qua, việc ứng dụng BIM tập trung vào sử dụng phần mềm BIM. Phần mềm BIM được đưa vào giảng dạy trong học phần Tin học chuyên ngành 2 bên cạnh phần mềm thông dụng là Autocad được giảng dạy trong học phần Tin học chuyên ngành 1 . Sau đó sinh viên được khuyến khích sử dụng phần mềm BIM vào các đồ án thiết kế kiến trúc.

- Tin học chuyên ngành 2: Nội dung chủ yếu là giới thiệu tổng quan về BIM và cách xây dựng, khai triển kỹ thuật và trình diễn thiết kế với mô hình công trình thông qua phần mềm BIM là Revit Architecture. 
- Đồ án kiến trúc: từ đồ án kiến trúc năm 3 trở đi và đồ án tốt nghiệp, sinh viên có thể sử dụng phần mềm Revit Architecture trong thực hiện đồ án.

Với hiệu quả mà Revit Architecture đem lại trong thực hiện đồ án cũng như nhu cầu nhân lực BIM ngày càng tăng từ các nhà tuyển dụng, tỷ lệ sinh viên lựa chọn phần mềm Revit Architecture để thực hiện đồ án đã tăng dần theo các khóa. Sau khi ra trường, tỷ lệ sinh viên làm việc trong các công ty ứng dụng BIM với yêu cầu sử dụng Revit Architecture cũng tăng.

Như vậy, việc ứng dụng BIM trong đào tạo ngành kiến trúc ở trường ĐHXD Miền Tây được áp dụng cho chương trình đào tạo chính quy, bậc đại học theo hình thức khóa học đơn ngành dành riêng cho sinh viên kiến trúc. Nội dung đào tạo tập trung vào sử dụng phần mềm BIM là Revit Architecture nhằm phát triển kỹ năng lập mô hình. Kỹ năng phân tích mô hình và kỹ năng cộng tác chưa được chú trọng. Tuy chỉ mới tập trung vào phát triển kỹ năng lập mô hình với BIM nhưng bước đầu cũng đã đem lại hiệu quả nhất định cho công tác đào tạo.

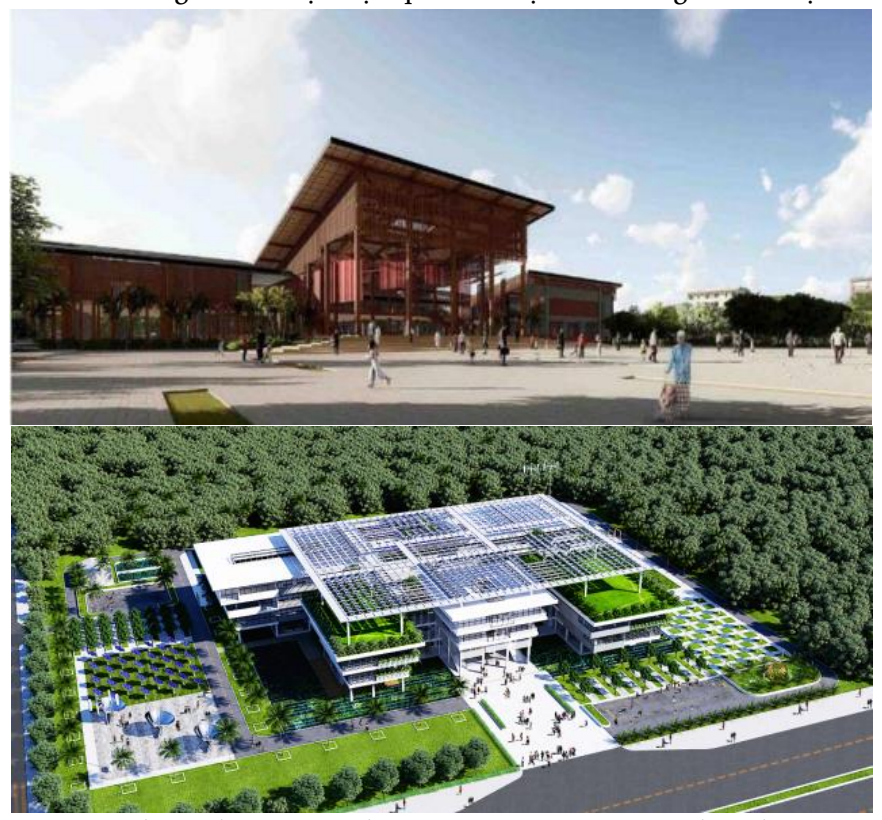

Hình 5. Đồ án tốt nghiệp kiến trúc sư thực hiện với phần mềm Revit Architeture (Giải thưởng Loa Thành 2018 \& 2019).

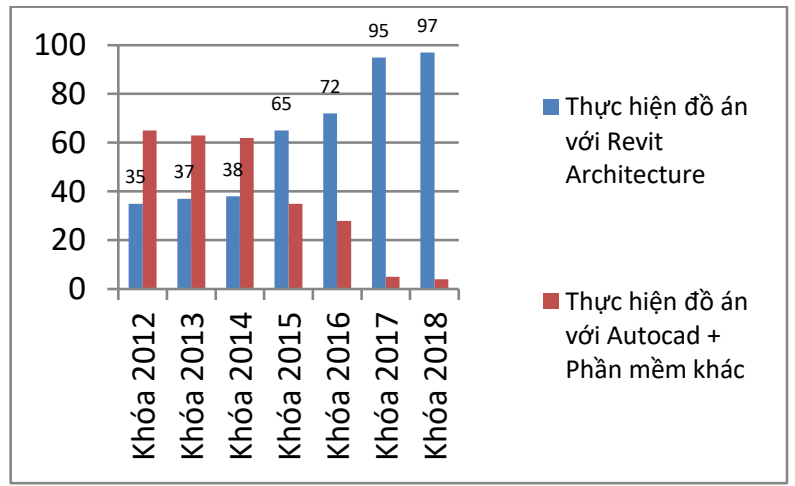

Hình 6. Tỷ lệ ứng dụng Revit Architecture trong thực hiện đồ án.

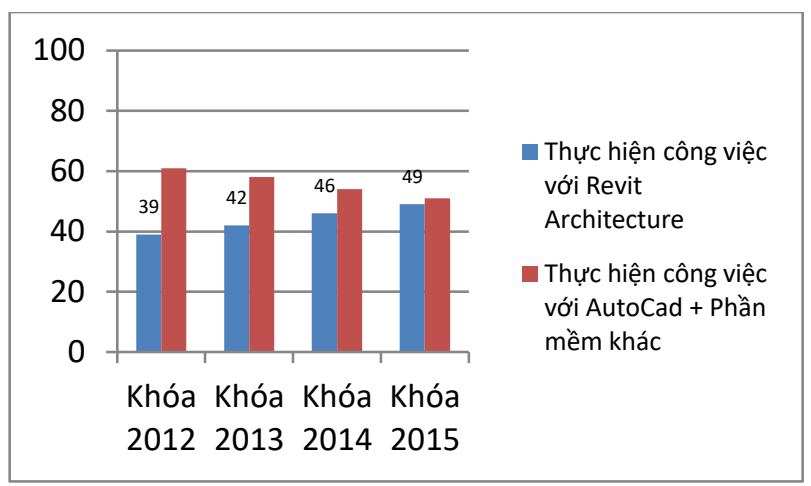

Hình 7. Tỷ lệ ứng dụng Revit Architecture trong công việc.

\subsection{Một số giải pháp nhằm đẩy mạnh đào tạo BIM cho sinh viên ngành} kiến trúc

Để đẩy mạnh hơn nữa việc ứng dụng BIM nhằm đáp ứng nhu cầu năng lực BIM ngày càng tăng của xã hội, chương trình đào tạo ngành kiến trúc được kiến nghị điều chỉnh, bổ sung như sau:

- Bổ sung thêm học phần Tin học chuyên ngành 3 để phát triển kỹ năng phân tích và cộng tác trên BIM cho sinh viên. Trong học phần này, sinh viên sẽ được hướng dẫn sử dụng các phần mềm như Climate Consultant, $\mathrm{CFD}$, Insight phân tích khu đất xây dựng và phân tích hiệu quả công trình để thiết kế công trình bền vững, công trình xanh. Đồng thời, sinh viên cũng được hướng dẫn thực hành thiết kế cộng tác một dự án nhỏ theo nhóm.

- Phân tích khu đất xây dựng và phân tich hiệu quả công trình trở thành nội dung bắt buộc trong thực hiện các đồ án năm $3,4,5$ và đồ án tốt nghiệp.

- Bổ sung đồ án Cấu tạo kiến trúc dưới dạng học phần tự chọn. Trong đồ án này, nhóm 3 - 5 sinh viên sẽ cùng phối hợp thực hiện khai triển kỹ thuật một công trình có quy mô từ 3-5 tầng để rèn luyện kỹ năng cộng tác trong cùng ngành.

Với những điều chỉnh bổ sung này, BIM sẽ được giảng dạy ở học phần Tin học chuyên ngành $2 \& 3$. Sinh viên sẽ ứng dụng BIM thực hiện các đồ án năm 3, 4, 5 và cùng phối hợp thực hiện đồ án Cấu tạo kiến trúc. Mục tiêu là nhằm phát triển kỹ năng lập mô hình, kỹ năng phân tích thiết kế và kỹ năng cộng tác đơn ngành. Trong điều kiện hiện tại, vẫn chưa thể tổ chức cộng tác liên ngành cho sinh viên trong trường.

\section{Kết luận}

Việc thiếu nguồn nhân lực có khả năng đi theo khuynh hướng BIM là một trở ngại lớn trong việc áp dụng BIM hiện nay. Do đó việc ứng dụng BIM trong đào tạo ở các trường đại học ngành Xây dựng Kiến trúc là cần thiết, góp phần thúc đẩy quá trình triển khai BIM ở Việt Nam.

Theo kinh nghiệm từ các trường đại học trên thế giới, BIM được đưa vào chương trình đào tạo theo nhiều phương pháp và hình thức khác nhau. Để ứng dụng BIM, trước hết cần phân tích, đánh giá lại 
chương trình đào tạo của ngành, điều kiện đội ngũ giảng viên, cơ sở vật chất của trường. Từ đó, đề xuất các giải pháp điều chỉnh, cập nhật chương trình đào tạo, điều chỉnh lại nội dung một số học phần, bổ sung những học phần mới hoặc xây dựng chương trình đào tạo mới với BIM là nội dung chủ đạo.

Một số giải pháp được đề xuất nhằm đẩy mạnh đào tạo BIM cho sinh viên ngành kiến trúc tại trường ĐHXD Miền Tây tập trung vào phát triển kỹ năng lập mô hình, kỹ năng phân tích mô hình và kỹ năng cộng tác đơn ngành. Do đó, cần tiếp tục nghiên cứu sự phối hợp liên ngành trong trong quá trình đào tạo để sinh viên có kiến thức và kỹ năng về phối hợp, cộng tác liên ngành trong quá trình làm việc theo BIM.\

\section{Tài liệu tham khảo}

[1]. International Organization for Standardization (ISO, 2018). BS EN ISO 19650-1, "Organization and digitization of information about buildings and civil engineering works, including building information modelling(BIM) Information management using building information modelling - Part1: Concepts and principles".

[2]. Eddy Krygiel and Bradley Nies, Green BIM: Successful Sustainable Design with Building Information Modeling. Wiley Publishing, Inc., 2008.

[3]. Barison, M. B., \& Santos, E. T, "BIM Teaching Strategies: an Overview of Current Approaches”, 2010. [Trực tuyến]. Địa chỉ: http://www.uel.br/pessoal/barison/Artigos_Tese/p288.pdf

[4]. NATSPEC, "BIM education - Global - 2021", International Construction Information Society. [Trực tuyến]. Địa chỉ: https://www.icis.org/wpcontent/uploads/2021/05/BIM-Education-Global-2021-Update-ReportV8.0.pdf

[5]. Zulfikar A. Adamu, Tony Thorpe, "How universities are teaching BIM: a review and case study from the UK". Journal of Information Technology in Construction, 2016. http://www.itcon.org/2016/8

[6]. "Nghiên cứu xây dựng tài liệu đào tạo phục vụ xây dựng nguồn nhân lực BIM cho ngành xây dựng Việt Nam”, Tạp chí kinh tế xây dựng số 4/2020.

[7]. Bộ Xây dựng, Công bố chương trình khung đào tạo, bồi dưỡng áp dụng Mô hình thông tin công trình (BIM) trong giai đoạn thí điểm, 1056/QĐ-BXD, 2017.

[8]. Barison, M. B., \& Santos, E. T, "Review and Analysis of Current Strategies for Planning a BIM Curriculum”, 2010. [Trực tuyến]. Địa chỉ: https://citeseerx.ist.psu.edu/viewdoc/download?doi $=10.1 .1 .453 .3898 \& \mathrm{r}$ ep $=$ rep1\&type $=$ pdf

[9]. Anas . Bataw, "On the Integration of Building Information Modelling in Undergraduate Civil Engineering Programmes in the United Kingdom”, 2016. [Trực tuyến]. Địa chỉ: https://www.research.manchester.ac.uk/portal/files/54582440/FULL_TE XT.PDF

[10]. The Higher Education Academy, "Embedding Building Information Modelling (BIM) within the taught curriculum", 2013. [Trực tuyến]. Địa chỉ: https://www.heacademy.ac.uk/system/files/BIM_June2013.pdf

[11]. Yasser Razgan Almutiri, "Empirical Investigation into Development of a curricular Framework to Embed Building Information Modelling with Undergraduate Architectural Programmes within Saudi Arabia", 2016. [Trực tuyến]. Địa chỉ: http://usir.salford.ac.uk/id/eprint/38770/ 